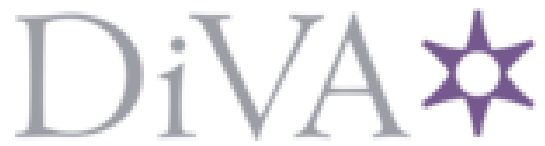

http://www.diva-portal.org

Preprint

This is the submitted version of a paper presented at 12th IAPR International Conference on Biometrics, Crete, Greece, June 4-7, 2019.

Citation for the original published paper:

Hernandez-Diaz, K., Alonso-Fernandez, F., Bigun, J. (2019)

Cross Spectral Periocular Matching using ResNet Features

In: 2019 International Conference on Biometrics (ICB)

Biometrics (ICB), IAPR International Conference on

https://doi.org/10.1109/ICB45273.2019.8987303

N.B. When citing this work, cite the original published paper.

Permanent link to this version:

http://urn.kb.se/resolve?urn=urn:nbn:se:hh:diva-40499 


\title{
Cross Spectral Periocular Matching using ResNet Features
}

\author{
Kevin Hernandez-Diaz, Fernando Alonso-Fernandez, Josef Bigun \\ Halmstad University \\ $\{$ kevher, feralo, josef.bigun $\}$ ahh. se
}

\begin{abstract}
Periocular recognition has gained attention in the last years thanks to its high discrimination capabilities in less constraint scenarios than other ocular modalities. In this paper we propose a method for periocular verification under different light spectra using CNN features with the particularity that the network has not been trained for this purpose. We use a ResNet-101 pretrained model for the ImageNet Large Scale Visual Recognition Challenge to extract features from the IIITD Multispectral Periocular Database. At each layer the features are compared using $\chi^{2}$ distance and cosine similitude to carry on verification between images, achieving an improvement in the EER and accuracy at $1 \%$ FAR of up to $63.13 \%$ and $24.79 \%$ in comparison to previous works that employ the same database. In addition to this, we train a neural network to match the best CNN feature layer vector from each spectrum. With this procedure, we achieve improvements of up to 65\% (EER) and $87 \%$ (accuracy at 1\% FAR) in cross-spectral verification with respect to previous studies.
\end{abstract}

\section{Introduction}

In 2009 U. Park et al. [16] introduced the concept of periocular recognition. They described this area as the facial region in the immediate vicinity of the eye. It has raised as a promising biometric trait for its high discriminatory rate while having more relaxed acquisition requirements than its other ocular counterparts [20][14][22] as well as being resilient to aging [6] and expressions [15], which makes it ideal for non-cooperative scenarios [7]. Moreover, it can be used not only as a sole biometric trait but also in combination with others like iris or face in order to increase performance [18], if these are available.

Convolutional Neural Networks (CNNs) have gained a lot of popularity since in 2012 Krizhevsky et al. [9] won the ImageNet Large Scale Visual Recognition Challenge with AlexNet. They have become the state of the art in many pattern recognition applications [2], but since they rely on a huge amount of data to work properly, their use in biomet- ric systems are still somewhat limited, with work mostly focused on face [17], iris [3] and soft biometrics [23].

Based on the study of Nguyen et al. [11], the authors in Hernandez-Diaz et al. [5] investigated the use of features from pre-trained off-the-shelf CNNs for periocular recognition, eliminating the necessity of designing and training new CNNs. In the present work, we further investigate the behaviour of these pre-trained CNN architectures in crossspectral periocular scenarios. Cross-spectral comparison is gaining attention in iris [10] and face [12] recognition, as well as in periocular research [21]. The choice of a particular illumination is based on a trade-off between different factors, including the quality required or practical aspects, such as the use of near-infrared images in controlled conditions (e.g. border control) vs. visible images in uncontrolled environments (e.g. smartphones). It may also happen that images captured with one type of illumination need to be compared with legacy images captured in a different spectrum. Unfortunately, the performance of crossspectral matching is significantly degraded in comparison with matching of images captured in the same spectrum [21].

The rest of the paper is organized as follows. In Section 2, we present our experimental framework, including the database employed, the pre-processing steps, and the protocol employed for cross-spectral periocular recognition using off-the-shelf CNN features. Section 3 presents the results obtained, both in same-sensor and cross-sensor scenarios. Finally, conclusions are given in Section 4.

\section{Experimental Framework}

\subsection{Database and Preprocessing}

Periocular databases with data in different spectra are a scarce resource [1]. In this work we use the IIITD Mulstispectral Periocular database [22], the only database available that provides periocular images in three different spectra: Visible, Night Vision and Near Infrared. Visible images were captured using a Nikon SLR camera; Night vision images were taken with a Sony HandyCAM in night vision mode and for NIR images, they used a Cogent Iris scan- 


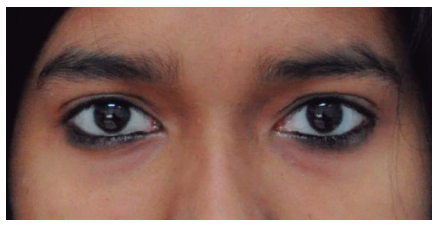

a)

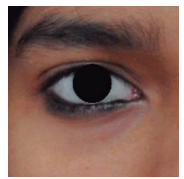

d)

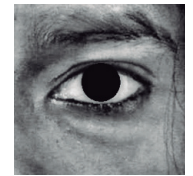

e)

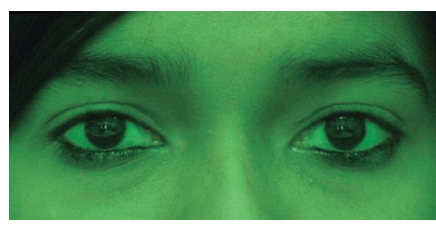

b)

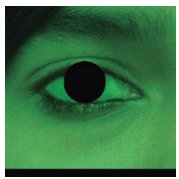

f)

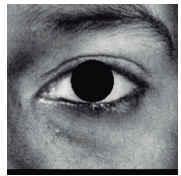

g)
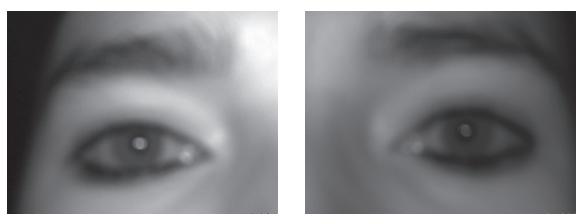

c)

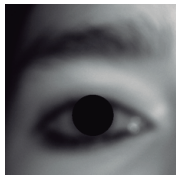

h)

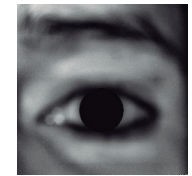

i)

Figure 1. samples of DB images: a) original visible spectrum image, b) original night vision image, c) original NIR images, d) centered, masked, scaled and cropped visible image, e) gray-scaled and contrast enhanced visible image, f) centered, masked, scaled and cropped night vision image, g) gray-scaled and contrast enhanced night vision image, h) masked and cropped NIR image, i) contrast enhanced NIR image

ner. Visible and Night Vision images were captured at a distance of 1.3 meters while NIR images were taken from 15 centimeters. All images were taken in a controlled environment, with proper illumination for Visible and NIR images and very low illumination for Night Vision images. Some examples are shown in Figure 1 (top)

The database contains images from 62 persons with 5 pictures per person in Visible and Night Vision spectrum containing both periocular areas, whereas in NIR there are 10 images per person, 5 for each eye. The resolution per spectrum are $601 \times 301,540 \times 260$ and $640 \times 480$ for Visible, Night Vision and NIR images respectively .

For this work, iris and sclera radius were manually annotated and irises masked to ensure periocular recognition. For Visible and Night Vision images, left and right eyes were centered, scaled to have their sclera radius equal to the mean of the database and cropped to be a square with a side length of 4 times the mean sclera radius. NIR images were first cropped to have a square shape with the same vertical length as the original image. Two different cases were considered for this step, if the sclera center fell close to the left or right border, the cropping was done so no extra padding was necessary, if the eye had enough separation to the borders, the cropping was made around the sclera center. No further normalization was done in order to avoid an excessive inclusion of artifacts or excessive cropping, this was due to the different acquisition technique used for this type of images.

In addition to this, extra preprocessing techniques were tried and compared to find the best case for same spectrum verification. For this purpose, all images were converted to gray-scale and contrast enhanced by Contrast-Limited Adaptive Histogram Equalization (CLAHE) [8] algorithm. We employ CLAHE since it is usually the preprocessing choice with images of the eye region [19].

Finally, since ResNet101 is configured to have as input RGB images of size $224 \times 224$, the pictures were resized and gray-scale images replicated to have three channels in order to fit the input size of the network. Figure 1 shows examples of pre and post processed images for each spectrum [19].

\subsection{Experimentation}

We carry out verification experiments for periocular images in different spectra, Visible, Night Vision and NIR with the method described in Hernandez-Diaz et al. [5] using the CNN that achieved best results. The CNN used for this paper is Matlab's ResNet-101 model [4]. The network consists of 101 convolutional layers trained on more than a million images from the ImageNet database to classify images within 1000 classes.

We fed the network with the post-processed images and extracted the output of every layer, including Convolution, $\mathrm{ReLu}$, Batch Normalization, Fully Connected and Addition layers as shown in the Figure 2. Then, for each layer, we tried a verification system that compares its output vectors using simple similitude measures as the ones shown in equations 1 and 2 . Equation 1 is a modified $\chi^{2}$ distance that uses the absolute value of the elements of the vectors, and normalize them so the sum of their elements equals 1 , this is done because this distance is usually applied to normalized histograms, whose entry values are always positive. Equation 2 is the cosine between vectors. The goal is to find the best performing layer for each case

$$
\chi^{2}=\sum_{i=1}^{n} \frac{\left(\left|x_{i}\right|-\left|y_{i}\right|\right)^{2}}{\left|x_{i}\right|+\left|y_{i}\right|}
$$




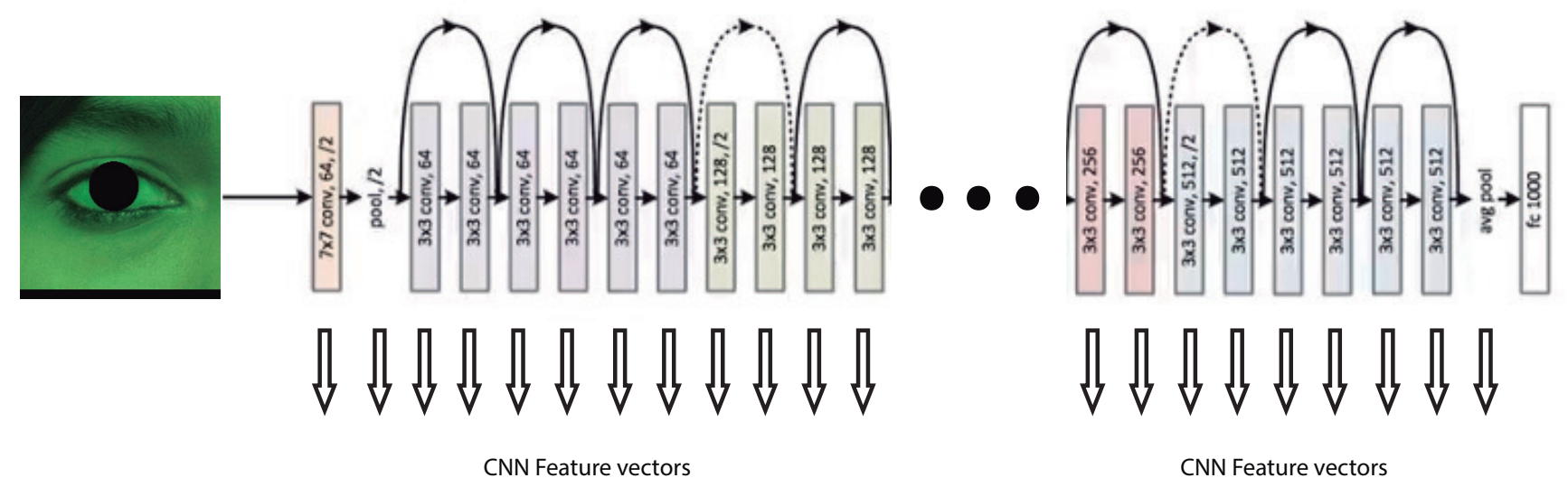

Figure 2. Extraction of the feature vectors from the CNN layers

$$
\cos (\alpha)=\frac{X^{T} * Y}{\|X\|\|Y\|}
$$

In our experiments, we consider each eye as a different user, thus having $62 \times 2=124$ users, and 5 images per user and spectrum. For genuine scores and same spectrum verification we compare all vectors of the same user using combination of two elements without repetitions, getting a total of 124 users $* 10$ combinations $=1240$ genuine scores. For the impostor score and same spectrum we compare the first image of every user with the second picture of all other users getting a total of $124 * 123=15252$ impostor scores.

For the genuine scores and cross-spectrum verification, we compared all images of each user in one spectrum against all images of the same user in the other spectrum, getting a total of 124 users $* 25$ combinations with a total of 3100 genuine scores. For the impostor scores we compare the first image of a user in one spectrum with the first image of the rest of the users in the other spectrum, getting a total of $124 * 123=15252$ impostor scores.

To further improve cross-sensor verification, we trained a neural network classifier that uses as input the feature vectors of the best CNN layer for each spectrum that we are comparing concatenated, and as output, the classification of either a genuine or an impostor matching. The network used in this research consists of an input layer of $2 *$ flattened CNN feature vector of size $50176=100352$ units, a hidden layer of 200 units with a ReLu activation function, another hidden layer with 20 units, a softmax layer and an output layer of size 2. We use SGDM, a minibatch size of 40 and the same amount of genuine and impostor vectors at every batch during the training process.

For neural network training, we used 5-fold crossvalidation. Each partition consists of $20 \%$ of the whole database, samples were randomly extracted and repetitions between partitions was not allowed. Moreover, instead of directly classifying the test cases at each test partition, we extract the softmax output, concatenate all tests partitions and manually change the threshold value at which we decide the vectors are genuine or impostor. This way we are not only able to calculate the EER and accuracy at $1 \%$ FAR, but we also get a more consistent behaviour of the neural network performance by considering all test partitions of each model as a whole.

\section{Results}

\subsection{Same Spectrum Verification}

First of all we carried out experiments with the features extracted at every layer of the CNN using $\chi^{2}$ distance and cosine similitude to find out at which layer the system perform best for same spectrum verification. We propose two different cases per spectrum: for Visible and Night Vision we used masked, centered, scaled and cropped periocular images from the originals but also gray-scaled images with its contrast enhanced by CLAHE algorithm. For NIR we used masked, cropped images but also tried contrast enhanced images. All this cases can be seen at Figure 1 (bottom).

\subsubsection{Similarity between vectors}

We report the EER at every layer of the CNN in the Figure 3 (top). Due to space constraints, we only give results of the best cases for each spectrum. For visible and Night Vision, they correspond to gray-scaled images with enhanced contrast. However, the best result in NIR is observed using original gray-scaled images without their contrast enhanced. It is worth noting that even though the CNN is trained using RGB images, the best results obtained are all for gray-scaled images. This can be due to fewer free variables in the input by having the same gray-scaled image 

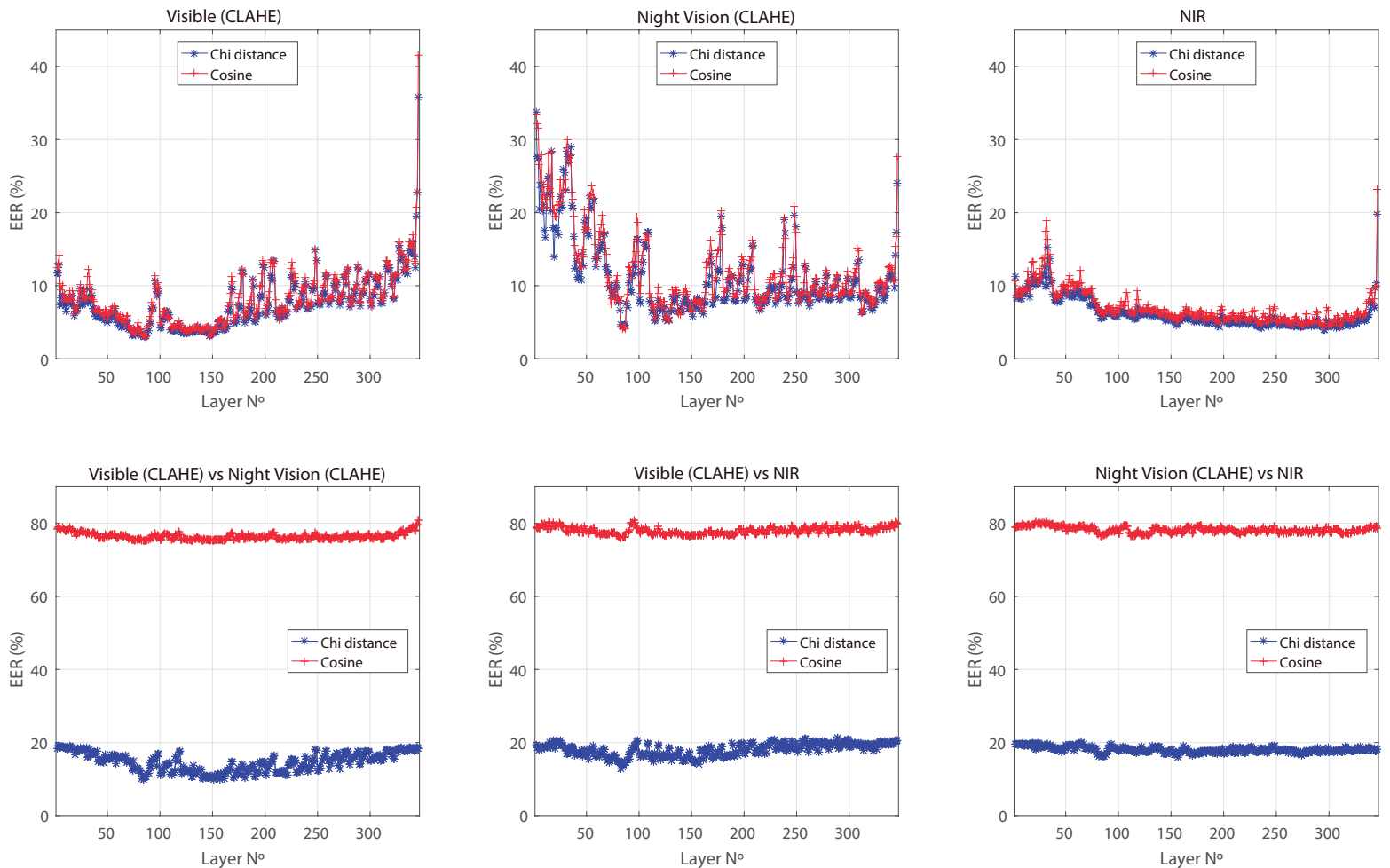

Figure 3. EER at every layer for same and cross spectral verification

replicated at every channel for a network that has not been trained for the task of periocular recognition.

From the results of Figure 3 (top), we can observe that the measures perform similar for each layer, with $\chi^{2}$ distance consistently giving slightly better results. We can also observe that each spectrum have different behaviour. Visible spectrum starts with a small EER and decrease more or less consistently until it reaches its minimum at layer 85 , with an EER of $2.95 \%$ using $\chi^{2}$ distance, having similar results until almost the first half of the network. Then, its behaviour become irregular, increasing and decreasing the EER every few layers. Night Vision has the most irregular performance of all three, with peaks all along the layers of up to more than $10 \%$ difference. It reaches the best performance at layer 84, with an EER of $4.04 \%$ using cosine similitude, as shown in the graph. Finally, NIR shows the most stable performance. Except for some peak at the beginning, it has a continuous descendent EER, reaching its minimum almost at the end of the network, at layer 295 with an EER of $3.97 \%$ using $\chi^{2}$ distance.

The best result for each spectrum is shown in Table 1, we give the EER at the best performing layer as well as the accuracy at $1 \%$ FAR. We also provide results of previous studies employing the same database [22]. It is worth noting that results shown in Table 1 were obtained using both periocular zones [22], which helps to improve the results during the verification process. Instead, here we consider each periocular zone as different users, which makes the recognition somewhat harder. By using the proposed method, we improve previously reported result by up to $63.13 \%$ and $24.79 \%$ for EER and accuracy at $1 \%$ FAR in Visible spectrum, respectively. In Night Vision spectrum, we improve results by up to $42.30 \%$ EER and up to $13.67 \%$ accuracy at $1 \%$ FAR. Finally, we increase accuracy results up to $1.65 \%$ in NIR spectrum.

\subsubsection{Neural Network}

Once we found the best performing layer for each spectrum, using these features, we seek to reduce the EER further by training a neural network capable of discerning genuine user vectors from impostor vectors in a verification scenario. The results for this experiments are shown in the Table 1 ( $5^{\text {th }}$ column, top). We have obtained the following EERs 5.08\% 3.95\% and 3.06\%, which improves results by $36.5 \%, 43.57 \%$ and $12.57 \%$ in relative terms for Visible, Night Vision and NIR respectively, in relation to those of Sharma et al. [22]. Achieving the best EER reported in this paper for Night Vision and NIR spectrum. Additionally, we 


\begin{tabular}{|c||c||c||c||c||c||c|}
\hline Spectrum & Type & $\chi^{2}$ & Cosine & Neural Network & best in [22] & improvement \\
\hline \hline \multirow{2}{*}{ Visible } & EER (\%) & $\mathbf{2 . 9 5}$ & 3.13 & 5.08 & $\sim 8.00$ & $\mathbf{- 6 3 . 1 3}$ \\
& Acc (\%) & $\mathbf{9 6 . 0 5}$ & 95.57 & 83.31 & 76.97 & $\mathbf{+ 2 4 . 7 9}$ \\
\hline \multirow{2}{*}{ Night } & EER (\%) & 4.48 & 4.04 & $\mathbf{3 . 9 5}$ & $\sim 7.00$ & $\mathbf{- 4 3 . 5 7}$ \\
& Acc (\%) & 88.23 & $\mathbf{9 0 . 0 0}$ & 89.35 & 79.18 & $\mathbf{+ 1 3 . 6 7}$ \\
\hline \multirow{2}{*}{ NIR } & EER (\%) & 3.97 & 4.41 & $\mathbf{3 . 0 6}$ & $\sim 3.5$ & $\mathbf{- 1 2 . 5 7}$ \\
& Acc (\%) & $\mathbf{9 4 . 0 3}$ & 93.47 & 89.72 & 92.50 & $\mathbf{+ 1 . 6 5}$ \\
\hline \hline \multirow{2}{*}{ VisiblevsNight } & EER (\%) & 9.79 & 75.33 & $\mathbf{5 . 1 3}$ & $\sim 9$ & $\mathbf{- 4 3 . 0 0}$ \\
& Acc (\%) & 51.19 & 8.42 & $\mathbf{8 8 . 1 9}$ & 71.93 & $\mathbf{+ 2 2 . 6 1}$ \\
\hline \multirow{2}{*}{ VisiblevsNIR } & EER (\%) & 12.88 & 76.08 & $\mathbf{5 . 1 9}$ & $\sim 15$ & $\mathbf{- 6 5 . 4 0}$ \\
& Acc (\%) & 29.00 & 3.35 & $\mathbf{8 8 . 1 3}$ & 47.08 & $\mathbf{+ 8 7 . 1 9}$ \\
\hline \multirow{2}{*}{ NightvsNIR } & EER (\%) & 15.95 & 76.53 & $\mathbf{1 0 . 1 9}$ & $\sim 15$ & $\mathbf{- 3 2 . 0 7}$ \\
& Acc (\%) & 23.74 & 2.58 & $\mathbf{8 1 . 5 5}$ & 48.21 & $\mathbf{+ 6 9 . 1 6}$ \\
\hline
\end{tabular}

Table 1. Summary of results. The results shown in the columns $3^{\text {rd }}$ to $5^{\text {th }}$ are obtained considering each eye as a different user, while the results of column $6^{\text {th }}$ used both eyes for matching. The column improvement shows the change of the best case (marked in bold) with respect to the results of [22]

improve the accuracy at 1\% FAR for Visible and Night Vision by $8.23 \%, 12.84 \%$ with respect to [22].

We present the behaviour of the neural network systems from which we obtained the best results shown in Table 1 in the DET graph in Figure 4. Here we can appreciate the similar behaviour of the Visible and Night spectra, with Night performing better all along the curve. The NIR curve on the other hand, have a higher slope than the other two, which gives a better EER but a worse accuracy at low FAR.

\subsection{Cross-Spectral Matching}

For cross-spectral matching, we follow the same procedure as for same-spectrum, with the difference that we only consider gray-scaled images to reduce differences in the RGB channels.

\subsubsection{Similarity between vectors}

We report the EER at every layer for each cross-spectral case (Visible and Night Vision, Visible and NIR, Night Vision and NIR) in the Figure 3 (bottom). The first thing that we notice is that the cosine similitude performs really bad in comparison with $\chi^{2}$ distance and previous results for same spectrum experiments. This can be due to an increase in sign discrepancies at the feature vectors extracted which $\chi^{2}$ distance can be more resilient to.

For cross-spectrum between visible and night vision images, we observe that the minimum is achieved at layer 157 with an EER of $9.79 \%$. For Visible vs. NIR images, the difference between the maximum and minimum EER is smaller, achieving the best EER at layer 83 with an EER of $12.88 \%$. Lastly, in Night and NIR comparison we observe a very steady function. It represents the case with the least amount of variation along all the layers, but also the one performing the worst, with an EER of 15.95 achieved at layer 157.

The best result for each cross-spectral case is shown in Table 1. Contrarily to same-spectrum experiments, here we only outperform the EER for Visible vs. NIR matching by reducing the error in $14,13 \%$ relatively to [22].

\subsubsection{Neural Networks}

In this section, we trained a neural network to distinguish between genuine and impostor users by using feature vectors extracted for different spectrum images. We made use of the best performance layers for each spectrum reported previously in section 3.1.1. (being layers 85, 84 and 295 for visible, night vision and NIR spectrum respectively). In contrast to same-spectrum experiments, in this case we fixed the spectrum order when we concatenated the vectors, this was due to the vectors differing not only in spectrum but also in the layer at which they were obtained, which contains useful information for the network that we may jeopardize by not fixing the order at which the vectors are presented.

We report the results of this section in the Table $1\left(5^{\text {th }}\right.$ col, bottom). We outperform any previous results found for every cross-matching scenario up to $65.40 \%$ in EER and $87.19 \%$ increase in accuracy at $1 \%$ FAR for Visible vs NIR. We also show the behaviour of those systems with their DET curves in Figure 4. Here we can observe the very similar behaviour of the Visible vs. Night and Visible vs. NIR systems. However what it is most remarkable is the high accuracy even at $0.1 \%$ FAR for all three systems, with an accuracy of $75.68 \%, 76.03 \%$ and $72,24 \%$ for Visible vs. Night, Visible vs. NIR and Night vs. NIR respectively. This means an improvement of up to $61.49 \%$ in accuracy for Visible vs. NIR matching with a system 10 times more secure. 


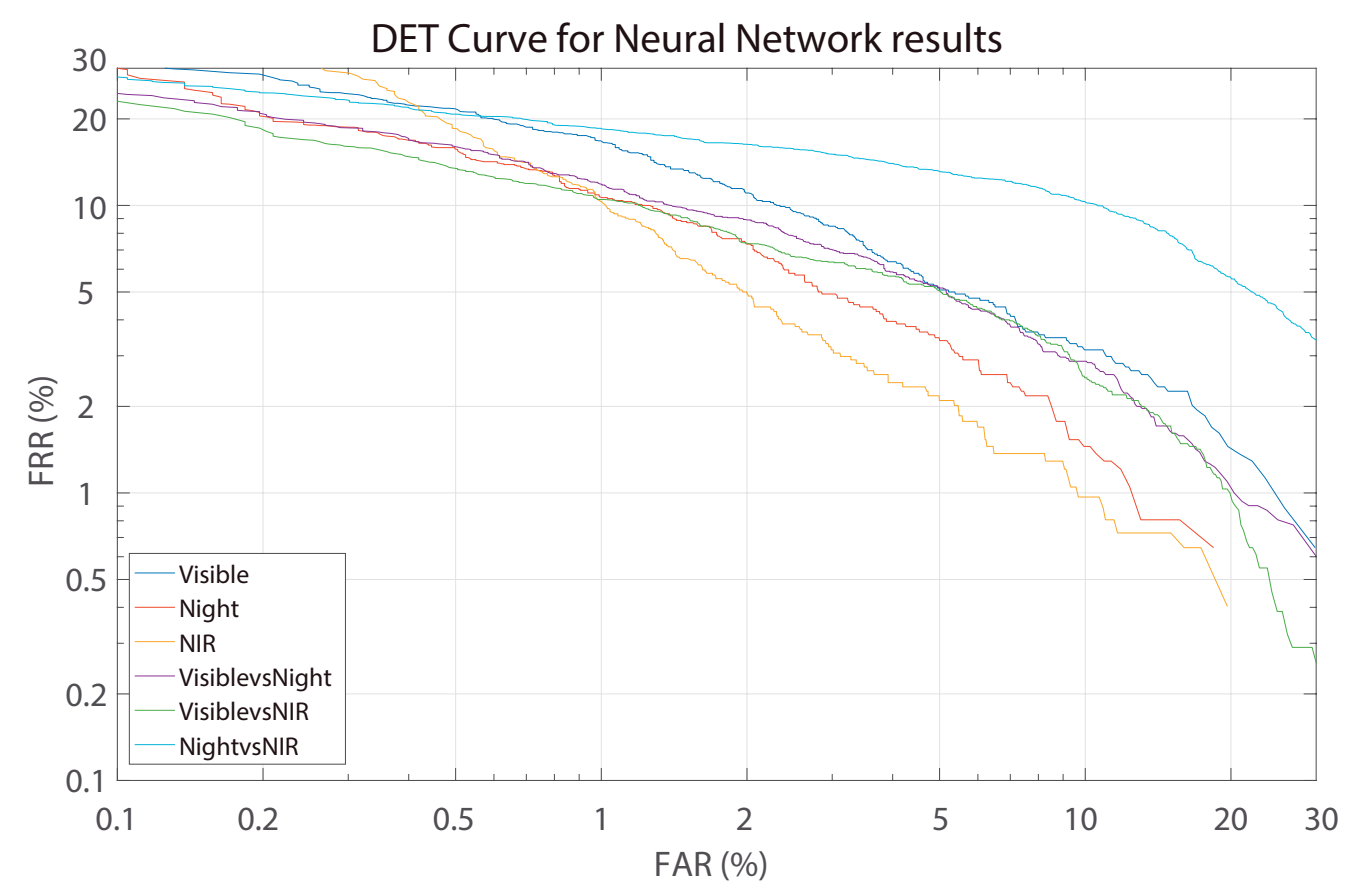

Figure 4. DET curve for Neural Network systems reported in Table 1 ( $5^{\text {th }}$ column)

\section{Conclusions}

Periocular recognition is an area of research that has a lot of potential still waiting to be fully exploited. It offers surprising accuracy rates in less constraint scenarios than its others ocular counterparts [1]. However, comparing ocular images captured in different spectra is usually much worse than if images were captured in the same spectrum. It is for this reason that developing methods capable of cross-spectral comparison holds a significant practical value, especially in periocular biometrics [13]. This is because the periocular region appears both in iris and face images, which are traditionally captured in the NIR and visible spectra, respectively.

In this work we investigate a new method for crosssensor periocular biometrics that makes use of the power of deep neural networks as feature extractor without having to train them, which is a huge drawback for most biometric databases for their rather small sizes. It does not only improve recognition in different spectra, but also provides great cross-spectral matching results. In this research we make use of the IIITD Multispectral Periocular Database [22] to carry on verification experimentation for periocular recognition in three different spectra: Visible, Night Vision and Near Infra Red, as well as cross-spectral verification between them. We use the widely known $\mathrm{CNN}$ architecture ResNet [4], pretrained with the ImageNet database and compare the features extracted at each layer using simple similitude measures that do not require any training, such as $\chi^{2}$ distance and cosine similitude between vectors. Furthermore, we train a neural network to improve the results obtained with the previous method.

We outperform the best results found for every category, more over, we achieve it in less favorable scenarios than those in which they were reported. In contrast to the others works found, we masked the irises to ensure pure periocular recognition and also consider each eye as a different user in the system, instead of using both eyes simultaneously to recognize a specific person. We improve EER up to $63.13 \%$ and accuracy at $1 \%$ FAR up to $24.79 \%$ for same spectrum recognition and up to $65.40 \%$ in EER and $87.19 \%$ accuracy at $1 \%$ FAR for cross-spectral matching. Moreover, we increase accuracy for cross-spectral scenarios up to $61.49 \%$ even if we consider a system 10 times more secure.

Future works include to try other famous CNN architectures e.g. [5] or our own, as well as explore cross-sensor comparison with other biometric modalities such as iris or face.

\section{References}

[1] F. Alonso-Fernandez and J. Bigun. A survey on periocular biometrics research. Pattern Recognition Letters, 82:92-105, 2016.

[2] Z. Fadlullah, F. Tang, B. Mao, N. Kato, O. Akashi, T. Inoue, and K. Mizutani. State-of-the-art deep learning: Evolving machine intelligence toward tomorrows intelligent network 
traffic control systems. IEEE Communications Surveys \& Tutorials, 19(4):2432-2455, 2017.

[3] A. Gangwar and A. Joshi. Deepirisnet: Deep iris representation with applications in iris recognition and cross-sensor iris recognition. In Image Processing (ICIP), 2016 IEEE International Conference on, pages 2301-2305. IEEE, 2016.

[4] K. He, X. Zhang, S. Ren, and J. Sun. Deep residual learning for image recognition. In Proceedings of the IEEE conference on computer vision and pattern recognition, pages 770-778, 2016.

[5] K. Hernandez-Diaz, F. Alonso-Fernandez, and J. Bigun. Periocular recognition using cnn features off-the-shelf. In 2018 International Conference of the Biometrics Special Interest Group (BIOSIG), pages 1-5. IEEE, 2018.

[6] F. Juefei-Xu, K. Luu, M. Savvides, T. D. Bui, and C. Y. Suen. Investigating age invariant face recognition based on periocular biometrics. In Biometrics (IJCB), 2011 International Joint Conference on, pages 1-7. IEEE, 2011.

[7] F. Juefei-Xu and M. Savvides. Unconstrained periocular biometric acquisition and recognition using cots ptz camera for uncooperative and non-cooperative subjects. In Applications of Computer Vision (WACV), 2012 IEEE Workshop on, pages 201-208. IEEE, 2012.

[8] K. Z. Karel. Graphics gems iv. chapter Contrast limited adaptive histogram equalization. Non-Uniform random variate generation, pages 474-485, 1994.

[9] A. Krizhevsky, I. Sutskever, and G. E. Hinton. Imagenet classification with deep convolutional neural networks. In Advances in neural information processing systems, pages 1097-1105, 2012.

[10] P. R. Nalla and A. Kumar. Toward more accurate iris recognition using cross-spectral matching. IEEE transactions on Image processing, 26(1):208-221, 2017.

[11] K. Nguyen, C. Fookes, A. Ross, and S. Sridharan. Iris recognition with off-the-shelf $\mathrm{cnn}$ features: A deep learning perspective. IEEE Access, 6:18848-18855, 2018.

[12] F. Nicolo and N. A. Schmid. Long range cross-spectral face recognition: matching swir against visible light images. IEEE Transactions on Information Forensics and Security, 7(6):1717-1726, 2012.

[13] I. Nigam, M. Vatsa, and R. Singh. Ocular biometrics: A survey of modalities and fusion approaches. Information $\mathrm{Fu}$ sion, 26:1-35, 2015.

[14] C. N. Padole and H. Proenca. Periocular recognition: Analysis of performance degradation factors. In Biometrics (ICB), 2012 5th IAPR International Conference on, pages 439-445. IEEE, 2012.

[15] U. Park, R. R. Jillela, A. Ross, and A. K. Jain. Periocular biometrics in the visible spectrum. IEEE Transactions on Information Forensics and Security, 6(1):96-106, 2011.

[16] U. Park, A. Ross, and A. K. Jain. Periocular biometrics in the visible spectrum: A feasibility study. In Biometrics: Theory, Applications, and Systems, 2009. BTAS'09. IEEE 3rd International Conference on, pages 1-6. IEEE, 2009.

[17] O. M. Parkhi, A. Vedaldi, A. Zisserman, et al. Deep face recognition. In $B M V C$, volume 1, page 6, 2015.
[18] K. B. Raja, R. Raghavendra, M. Stokkenes, and C. Busch. Multi-modal authentication system for smartphones using face, iris and periocular. In Biometrics (ICB), 2015 International Conference on, pages 143-150. IEEE, 2015.

[19] C. Rathgeb and A. Uhl. Secure iris recognition based on local intensity variations. In International Conference Image Analysis and Recognition, pages 266-275. Springer, 2010.

[20] G. Santos and H. Proença. Periocular biometrics: An emerging technology for unconstrained scenarios. In Computational Intelligence in Biometrics and Identity Management (CIBIM), 2013 IEEE Workshop on, pages 14-21. IEEE, 2013.

[21] A. F. Sequeira, L. Chen, J. Ferryman, P. Wild, F. AlonsoFernandez, J. Bigun, K. B. Raja, R. Raghavendra, C. Busch, T. Freitas Pereira, et al. Cross-eyed 2017: Cross-spectral iris/periocular recognition competition. In IEEE/IAPR International Joint Conference on Biometrics, IJCB, Denver, Colorado, USA, October 1-4, 2017, pages 725-732. IEEE, 2017.

[22] A. Sharma, S. Verma, M. Vatsa, and R. Singh. On cross spectral periocular recognition. In Image Processing (ICIP), 2014 IEEE International Conference on, pages 5007-5011. IEEE, 2014.

[23] J. Zhu, S. Liao, D. Yi, Z. Lei, and S. Z. Li. Multi-label cnn based pedestrian attribute learning for soft biometrics. In Biometrics (ICB), 2015 International Conference on, pages 535-540. IEEE, 2015. 